\title{
THREE-DIMENSIONAL EXPRESSION OF SEISMIC PARTICLE MOTIONS BY THE TRAJECTORY ELLIPSOID AND ITS APPLICATION TO THE SEISMIC DATA OBSERVED IN THE KANTO DISTRICT, JAPAN
}

\author{
Shozo Matsumura \\ National Research Center for Disaster Prevention, Ibaraki, Japan
}

(Received December 22, 1980; Revised June 10, 1981)

\begin{abstract}
A method for estimating an approximate three-dimensional seismic particle motion was developed. The characteristics of particle motions can be represented by three sets of eigenvectors and eigenvalues corresponding to the principal axes of a trajectory ellipsoid, which are obtained by diagonalizing the covariant tensor of the particle motions.

This method was applied to the initial portion of $\mathbf{P}$ and $\mathbf{S}$ waves observed at the Iwatsuki Crustal Activity Observatory, where the seismometers are installed at the bottom of a $3,500 \mathrm{~m}$ deep borehole. Difference in the particle motions between $\mathrm{P}$ and $\mathrm{S}$ waves was clearly expressed by the shape and the direction of the principal axes of the trajectory ellipsoid.

The shape of the trajectory ellipsoid reflects scattering of the wave in the medium, and the direction of the principal axes of that indicates the ray path of the wave. By examining regional distribution of the trajectory ellipsoids in the Kanto district, the following results were obtained: Scattering of seismic waves are mainly observed for the vertical component, and in a region north of $36^{\circ} \mathrm{N}$, seismic waves are scattered more intensely than in a region south of $36^{\circ} \mathrm{N}$. The distribution pattern of eigenvectors seems likely to indicate that the underground layers are inclined from the horizontal plane by about $10^{\circ}$ towards the north.
\end{abstract}

\section{Introduction}

Initial motions of seismic waves provide data useful for analyzing the incident direction of the waves. UTsU (1956), KURIHARA et al. (1974), and WADA and Nishimura (1971), who analyzed initial motions of $P$ waves, attributed the deviation pattern of the incident vectors to the local crustal structure. FuRUZAWA and IRIKURA (1970), and FURUZAWA (1974) used particle motions of seismic waves for distinguishing $P$ phases from $S$ phases. Their method, which observes the average pattern of particle motion, is so powerful that we can analyze even microearthquakes, of which the signals are usually too small for identifying the initial phases. It should be noted, however, that their treatment of the data is 
essentially two-dimensional although the vertical component is combined to them in actual analyses.

In an analysis of particle motions observed at the earth's surface or at the bottom of shallow wells, contribution of waves reflected at the earth's surface cannot be neglected, so that it becomes inadequate to treat all the componentstwo horizontals and one vertical-equally (NuTTLI, 1961), At the Iwatsuki Crustal Activity Observatory (IWT) of the National Research Center for Disaster Prevention, the observation of seismic waves is carried out at the bottom of a borehole which is as deep as $3,500 \mathrm{~m}$. If an appropriate time window is selected, therefore, the influence of reflected waves may be removed.

This paper deals with a new numerical procedure for representing the threedimensional particle motion by an ellipsoid. We refer to a characteristic ellipsoid which is called the trajectory ellipsoid. Then all the characteristics of particle motion can be parameterized by the three principal axes of the ellipsoid.

We apply this method to the data observed at IWT and discuss the physical meanings of the parameters obtained. Not only $\mathrm{P}$ waves but $\mathrm{S}$ waves are analyzed.

\section{Theory}

An example of particle motions for the initial $0.8 \mathrm{sec}$ of $\mathrm{P}$ wave is shown in Fig. 1, in which the locus is projected on three orthogonal planes. The sampling rate is $62.5 \mathrm{~Hz}$ and number of the sampled data is $N=50$. Such a projection is not quite adequate for bringing out the three-dimensional behaviour of particle motion very clearly, so that we introduce the trajectory ellipsoid which may approximately represent the particle motion. The principal axes of an ellipsoid is defined by the three orthogonal vectors. The square sum of projection of the particle trajectory takes on the extreme value in the direction of each vector. According to the Lagrange's unknown-parameter-method, a function

$$
f=\sum_{i=1}^{N}\left(a_{x} x_{i}+a_{y} y_{i}+a_{z} z_{i}\right)^{2}-\lambda^{2}\left(a_{x}{ }^{2}+a_{y}{ }^{2}+a_{z}{ }^{2}-1\right)
$$

is defined where $a=\left(a_{x}, a_{y}, a_{z}\right)$ is a unit vector, $x_{i}, y_{i}$ and $z_{i}$ the components of the particle motion, and $\lambda^{2}$ an unknown parameter, respectively. Putting the partial differentials of $f$ by $a_{x}, a_{y}$ and $a_{z}$ to zero, we obtain

$$
\begin{aligned}
& \frac{\partial f}{\partial a_{x}}=2 \sum_{i=1}^{N} x_{i}\left(a_{x} x_{i}+a_{y} y_{i}+a_{z} z_{i}\right)-2 \lambda^{2} a_{x}=0, \\
& \frac{\partial f}{\partial a_{y}}=2 \sum_{i=1}^{N} y_{i}\left(a_{x} x_{i}+a_{y} y_{i}+a_{z} z_{i}\right)-2 \lambda^{2} a_{y}=0, \\
& \frac{\partial f}{\partial a_{z}}=2 \sum_{i=1}^{N} z_{i}\left(a_{x} x_{i}+a_{y} y_{i}+a_{z} z_{i}\right)-2 \lambda^{2} a_{z}=0 .
\end{aligned}
$$

These equations are rewritten as

$$
E \boldsymbol{a}=\lambda^{2} \boldsymbol{a}
$$



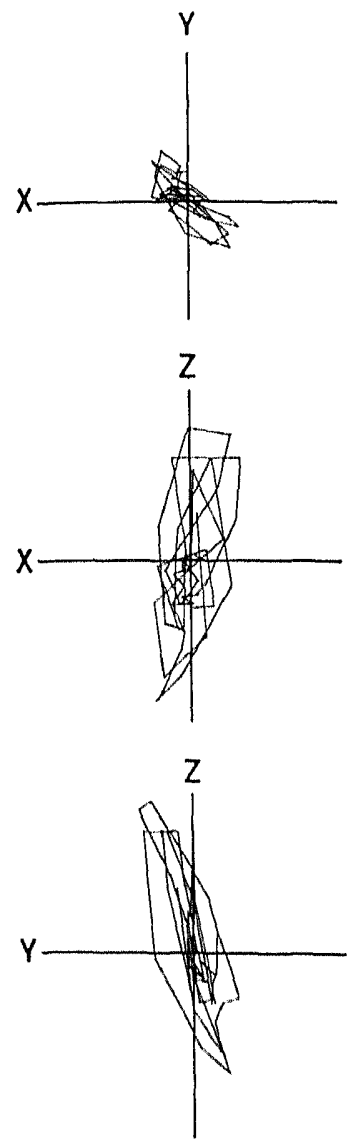

Fig. 1. An example of a particle motion projected on three orthogonal planes. The initial $0.8 \mathrm{sec}$ portion of the $P$ wave is shown with a sampling frequency of $62.5 \mathrm{~Hz}$.

$$
E=\sum_{i=n 1}^{N}\left(\begin{array}{ccc}
x_{i} x_{i} & x_{i} y_{i} & x_{i} z_{i} \\
y_{i} x_{i} & y_{i} y_{i} & y_{i} z_{i} \\
z_{i} x_{i} & z_{i} y_{i} & z_{i} z_{i}
\end{array}\right)
$$

The covariant tensor $E$ defines a tensor ellipsoid, where the eigenvalues and the eigenvectors are $\left(\lambda_{1}{ }^{2}, a_{1}\right),\left(\lambda_{2}{ }^{2}, a_{2}\right)$ and $\left(\lambda_{3}{ }^{2}, a_{3}\right)$. Assuming that $\lambda_{1}{ }^{2} \geqq \lambda_{2}{ }^{2} \geqq \lambda_{3}{ }^{2}$, the vectors defined by $\lambda_{1} a_{1}, \lambda_{2} a_{2}$ and $\lambda_{3} a_{3}$ identify the maximum, intermediate and minimum axes of the ellipsoid which represents the particle motion, respectively (see Fig. 2). Aspect ratios $\varepsilon_{1}=\lambda_{2} / \lambda_{1}$ and $\varepsilon_{2}=\lambda_{3} / \lambda_{1}$ are defined by the ratios of their magnitudes. If the wave is completely polarized, they are equal to zero. For the particle motion observed with a velocity seismometer, $\varepsilon_{1}{ }^{2}$ and $\varepsilon_{2}{ }^{2}$ represent distribution ratios of the wave energy for the three orthogonal directions. In the case of microearthquakes, it is necessary to remove contributions from environmental noises from the data. Observed amplitude $x_{i}$ is decomposed into seismic signal amplitude $X_{i}$ and noise amplitude $u_{i}$, so that we obtain $x_{i}=X_{i}+u_{i}$. 


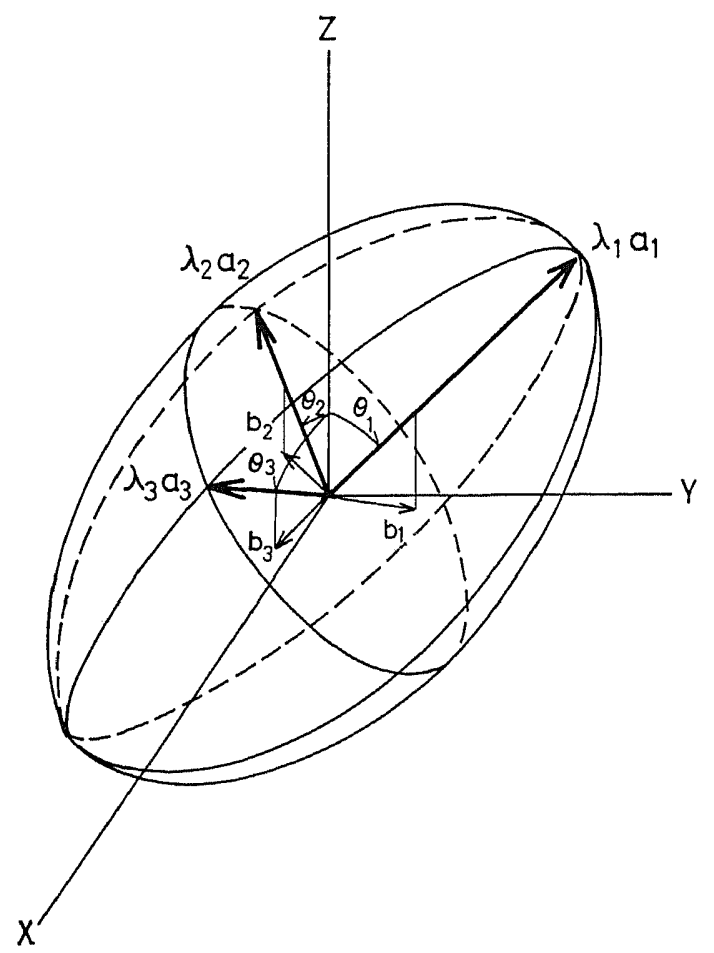

Fig. 2. A trajectory ellipsoid model expressing a particle motion. The vectors $\lambda_{1} a_{1}$, $\lambda_{2} a_{2}$ and $\lambda_{3} a_{3}$ are the maximum, the intermediate and the minimum principal axes of the ellipsoid, and $\theta_{1}, \theta_{2}$ and $\theta_{3}$ are their zenith angles. The vectors $b_{1}, b_{2}$ and $b_{3}$ are horizontal projections of $a_{1}, a_{2}$ and $a_{3}$, respectively.

Similarly, we have $y_{i}=Y_{i}+v_{i}$ and $z_{i}=Z_{i}+w_{i}$. Then we have

$$
\sum_{i=1}^{N} x_{i} y_{i}=\sum_{i=1}^{N} X_{i} Y_{i}+\sum_{i=1}^{N} X_{i} v_{i}+\sum_{i=1}^{N} Y_{i} u_{i}+\sum_{i=1}^{N} u_{i} v_{i}
$$

The second and the third terms of Eq. 5 reduce to zero because there is no correlation between seismic signals and environmental noises. Therefore, component $\sum_{i=1}^{N} x_{i} y_{i}$ in Eq. 4 is replaced by $\sum_{i=1}^{N} X_{i} Y_{i}=\sum_{i=1}^{N} x_{i} y_{i}-\sum_{i=1}^{N} u_{i} v_{i}$, and the other components are also replaced by terms in a similar manner. In an actual calculation, $\sum_{i=1}^{N} u_{i} v_{i}$ and others are estimated from noises neighboring the seismic signals on a seismic record.

\section{Data}

We used the data observed with the highly sensitive seismometers of velocity 


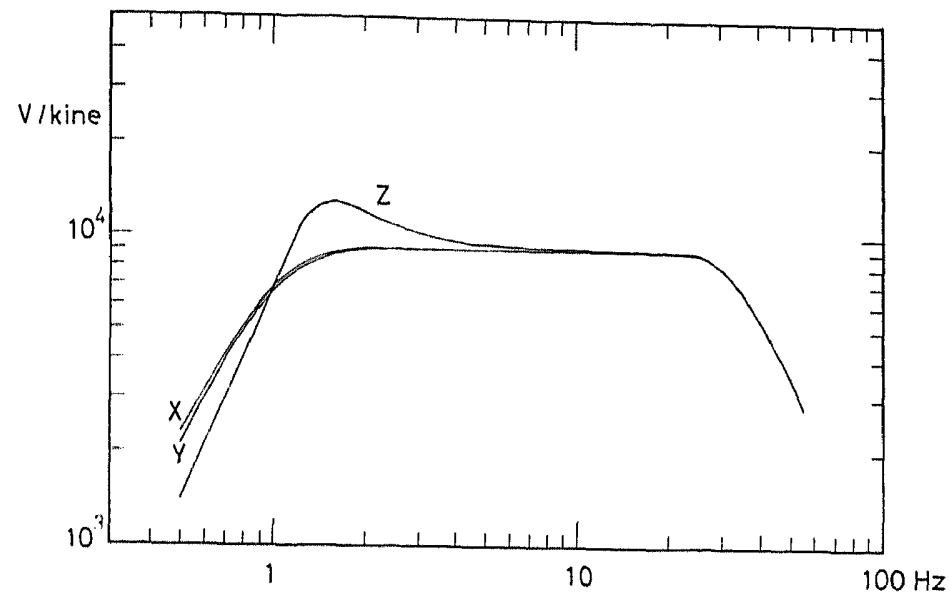

Fig. 3. Overall frequency responses of the observation system including the TEAC $\mathrm{R}-510$ magnetic-tape recorder. $X, Y$, horizontal; $Z$, vertical.

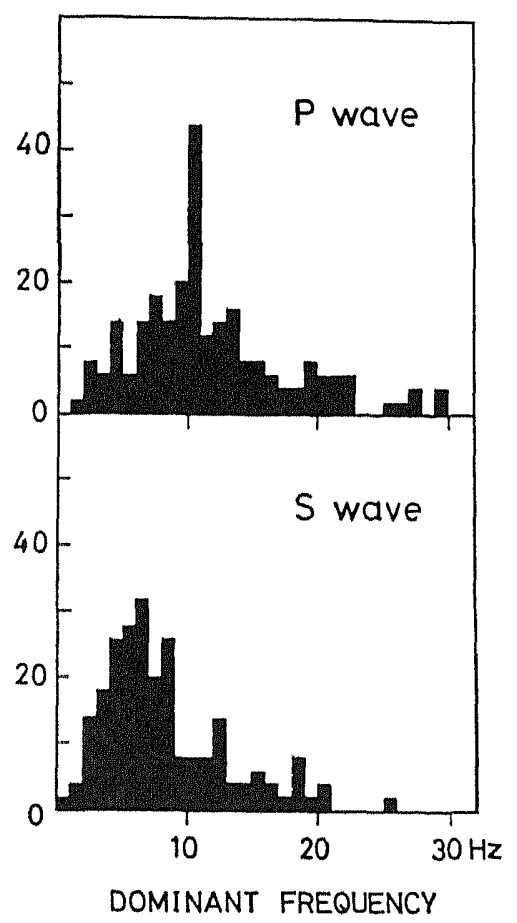

Fig. 4. Histograms of dominant frequencies of $\mathrm{P}$ and $\mathrm{S}$ waves.

type at IWT $\left(35.9258^{\circ} \mathrm{N}, 138.7381^{\circ} \mathrm{E}\right)$. The seismometers are installed at the bottom of a borehole of $3,500 \mathrm{~m}$ in depth. It is thus possible to obtain excellent data which are not contaminated by waves reflected from the earth's surface for the initial periods up to 2.5 and $6.3 \mathrm{sec}$ respectively for $\mathrm{P}$ and $\mathrm{S}$ waves (YAMAMIzU 
and GoTo, 1978). The characteristic sensitivity of the seismometers are shown in Fig. 3 for the three components. The data we used were taken from the magnetic-tape file of the observation in May 1976. The number of earthquakes which were used for the analysis amounts to 128 for $\mathrm{P}$ waves and 122 for $\mathrm{S}$ waves, respectively. The magnitude of earthquakes ranges $2.0-3.5$ for the $90 \%$ of the earthquakes studied. The hypocenters and magnitudes were determined by K. Tsumura of the Earthquake Research Institute of the University of Tokyo.

Seismic waves recorded in the magnetic tape were digitized at a sampling frequency of $62.5 \mathrm{~Hz}$. The data of three components were simultaneously sampled by an $\mathrm{AD}$ converter in order that no distortion of the trajectory ellipsoid caused by phase difference between the components arises. The width of time window $T$ must carefully be selected in order to analyze the initial phase of seismic waves. For approximating the particle motion by an ellipsoid, at least one cycle of the oscillating motion must be included in the sampled data. Dominant frequencies of both $P$ and $S$ waves were estimated and their histograms were shown in Fig. 4. Most of these frequencies are higher than $2.5 \mathrm{~Hz}$, so that $0.4 \mathrm{sec}$ is the lower limit for $T$. On the other hand, if $T$ is too long, the data sampled necessarily contain not only the initial phase but also the later phases, especially the one reflected from the surface. At the bottom of the deep borehole at IWT, the delay time of the reflected $P$ phase behind the initial phase is estimated as $2.5 \mathrm{sec}$. It should be the upper limit for $T$. Taking the lower and the upper limits of $T$ discussed above into consideration, we selected three time windows, say $T=0.4,0.8$ and $1.6 \mathrm{sec}$.

\section{Results and Discussion}

\subsection{P wave}

In the case of $P$ waves, principal axes of the trajectory ellipsoid were computed for 128 earthquakes. Mean values and standard deviations of aspect ratios $\varepsilon_{1}$ and $\varepsilon_{2}$ for $T=0.4,0.8$ and $1.6 \mathrm{sec}$ are listed in Table 1 . If the incident wave is a pure $\mathrm{P}$ wave, the aspect ratio should be zero, i.e., the shape of the ellipsoid looks like a needle. But the actual data given in Table 1 show that the ellipsoid has some thickness, which increases as the length of time window increases. SATo and MATSUMURA (1980) proved that the coda part of seismic waves is formed by scattered waves and that the energy ratio of transverse component to radial one increases as the time lag from the onset time of the phase increases, the ratio tending to 1.0 at the infinity of time. Such a result seems likely to explain the observed phenomena described above.

In the following discussion we shall mainly study the result obtained by using time window $T=0.8 \mathrm{sec}$ because it appears that other selections of time window lead to more or less a similar conclusion. Amplitude ratio of the seismic signal to noises is 2.0 at the minimum and 40 at the maximum, but no correlation between wave amplitudes and aspect ratios was found. On the other hand a characteristic 
Table 1. Mean values and standard deviations of aspect ratios $\varepsilon_{1}$ and $\varepsilon_{2}$ of trajectory ellipsoids which approximate the P- and S-particle motions for time windows of $0.4,0.8$ and $1.6 \mathrm{sec}$.

\begin{tabular}{cccc}
\hline & $0.4 \mathrm{sec}$ & $0.8 \mathrm{sec}$ & $1.6 \mathrm{sec}$ \\
\hline$\varepsilon_{1}(\mathrm{P})$ & $0.51 \pm 0.18$ & $0.58 \pm 0.15$ & $0.68 \pm 0.14$ \\
$\varepsilon_{2}(\mathrm{P})$ & $0.23 \pm 0.13$ & $0.30 \pm 0.12$ & $0.39 \pm 0.12$ \\
$\varepsilon_{1}(\mathrm{~S})$ & $0.68 \pm 0.15$ & $0.71 \pm 0.15$ & $0.76 \pm 0.13$ \\
$\varepsilon_{2}(\mathrm{~S})$ & $0.44 \pm 0.13$ & $0.48 \pm 0.15$ & $0.53 \pm 0.13$ \\
\hline
\end{tabular}

feature was found in regional distribution of the aspect ratios. The computed values of $\varepsilon_{1}$ and $s_{2}$ are classified into three categories, 0 to $m-2 / 3 \sigma, m-2 / 3 \sigma$ to $m+2 / 3 \sigma$ and $m+2 / 3 \sigma$ to 1 , where $m$ and $\sigma$ are the mean and the standard deviation given in Table 1. The regional distribution are shown in Figs. 5(a) and (b) with open circles, crosses and solid circles corresponding to the above three categories. Theoretically speaking, the aspect ratio representing the wave energy ratio of the scattered to the direct one depends only on the hypocentral distance provided the scatterings occur uniformly everywhere, because the ratio is uniquely determined as a function of time, which is normalized with respect to the S-P time. Therefore, the aspect ratio for a constant time window becomes larger for near earthquakes than for distant earthquakes since the corresponding width of the time window is relatively wide for near earthquakes. The regional distribution of $\varepsilon_{2}$ in Fig. 5(b) is harmonized with the above expectation, i.e., most of the epicenters characterized by an open circle (smaller aspect ratio) are located at distances from IWT larger than those characterized by a solid circle (larger aspect ratio).

Another systematic regional distribution is found for $\varepsilon_{1}$ in Fig. 5(a): larger $\varepsilon_{1}$ values in a region north of $36^{\circ} \mathrm{N}$ and smaller $\varepsilon_{1}$ values in a region south of $36^{\circ} \mathrm{N}$. These regions are bounded fairly clearly by a plane which is shown in the figure by hatching. The boundary strikes in E-W direction along the latitude line of $35.6^{\circ} \mathrm{N}$, and inclines about $40^{\circ}$ towards the north, which divides the Kanto district into two parts. It is suggested that the degree of wave scattering is higher in the region north of the boundary than in the region south of the boundary. TsusrURA (1973) reported that the spectral characteristic of microearthquakes in the Kanto district shows a systematic distribution with a distinct boundary along the line of $36^{\circ} \mathrm{N}$; the extent of higher frequencies contained in seismic waves from the region north of $36^{\circ} \mathrm{N}$ is larger than that from the region south of $36^{\circ} \mathrm{N}$. The distribution pattern presented in his paper is very similar to that of $\varepsilon_{1}$. These two results are compatible with one another if the intensity of scattering represented by $\varepsilon_{1}$ is assumed to be larger for high frequencies and smaller for low frequencies. The mean value of dominant frequencies of $\mathrm{P}$ waves is estimated to be $12.0 \mathrm{~Hz}$ from Fig. 4, but $13.8 \mathrm{~Hz}$ for those, of which $\varepsilon_{1}$ is more than $m+2 / 3 \sigma$, and $8.0 \mathrm{~Hz}$ for those, of which $\varepsilon_{1}$ is less than $m-2 / 3 \sigma$. Such differences in the mean frequency seems to support the assumption. 


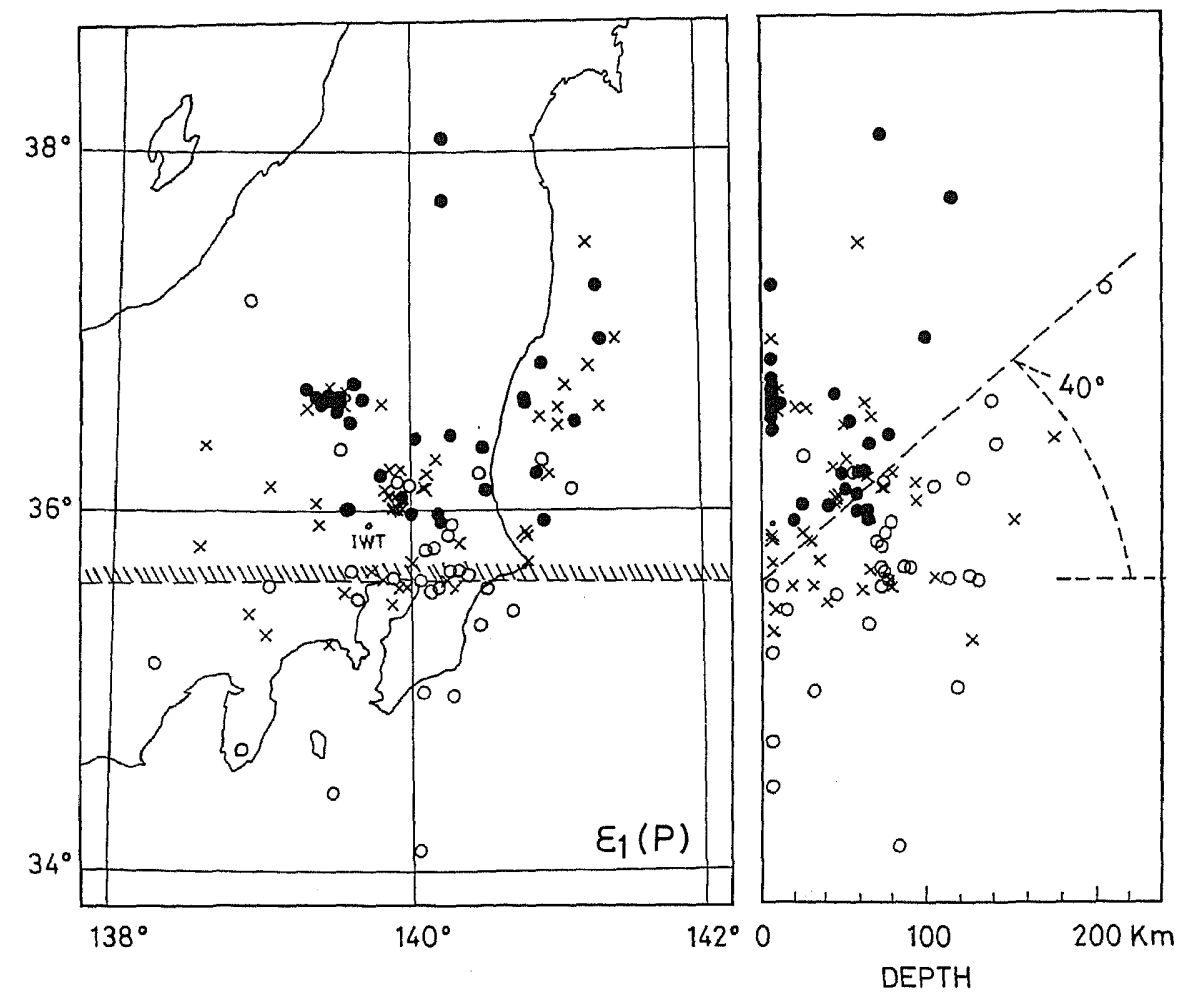

Fig. 5 (a)

Difference of scattering properties between those represented by $\varepsilon_{1}$ and $\varepsilon_{2}$ became clear after examining the direction of principal axes of ellipsoid. Figures $6(\mathrm{a}),(\mathrm{b})$ and (c) show vectors $b_{1}, b_{2}$ and $b_{3}$, respectively. These vectors are formed by projection of eigenvectors $a_{1}, a_{2}$ and $a_{3}$ on a horizontal plane. In these figures the origins of the horizontal vectors are moved from IWT to their epicenters. Arrowheads of the vectors are put so that they point upwards. As a general pattern, it is recognized that $b_{1}$ points towards IWT, $b_{2}$ against IWT and $\boldsymbol{b}_{3}$ is perpendicular to the direction of IWT. Vertical profiles of vectors $\boldsymbol{a}_{1}, \boldsymbol{a}_{2}$ and $\boldsymbol{a}_{3}$ can be expressed by their zenith angles $\theta_{1}, \theta_{2}$ and $\theta_{3}$ as defined in Fig. 2. In Fig. 7, $\theta_{1}, \theta_{2}$ and $\theta_{3}$ are plotted against $\theta$ which is the incident angle of a theoretical ray path calculated by using the MrKumo's velocity model (1966) as also shown in the figure. From these figures, following relations are found:

$$
\begin{aligned}
& \theta_{1} \risingdotseq \theta, \\
& \theta_{2} \risingdotseq 90^{\circ}-\theta, \\
& \theta_{3} \risingdotseq 90^{\circ} .
\end{aligned}
$$

With these figures and equations we can have a general picture of the shape and 

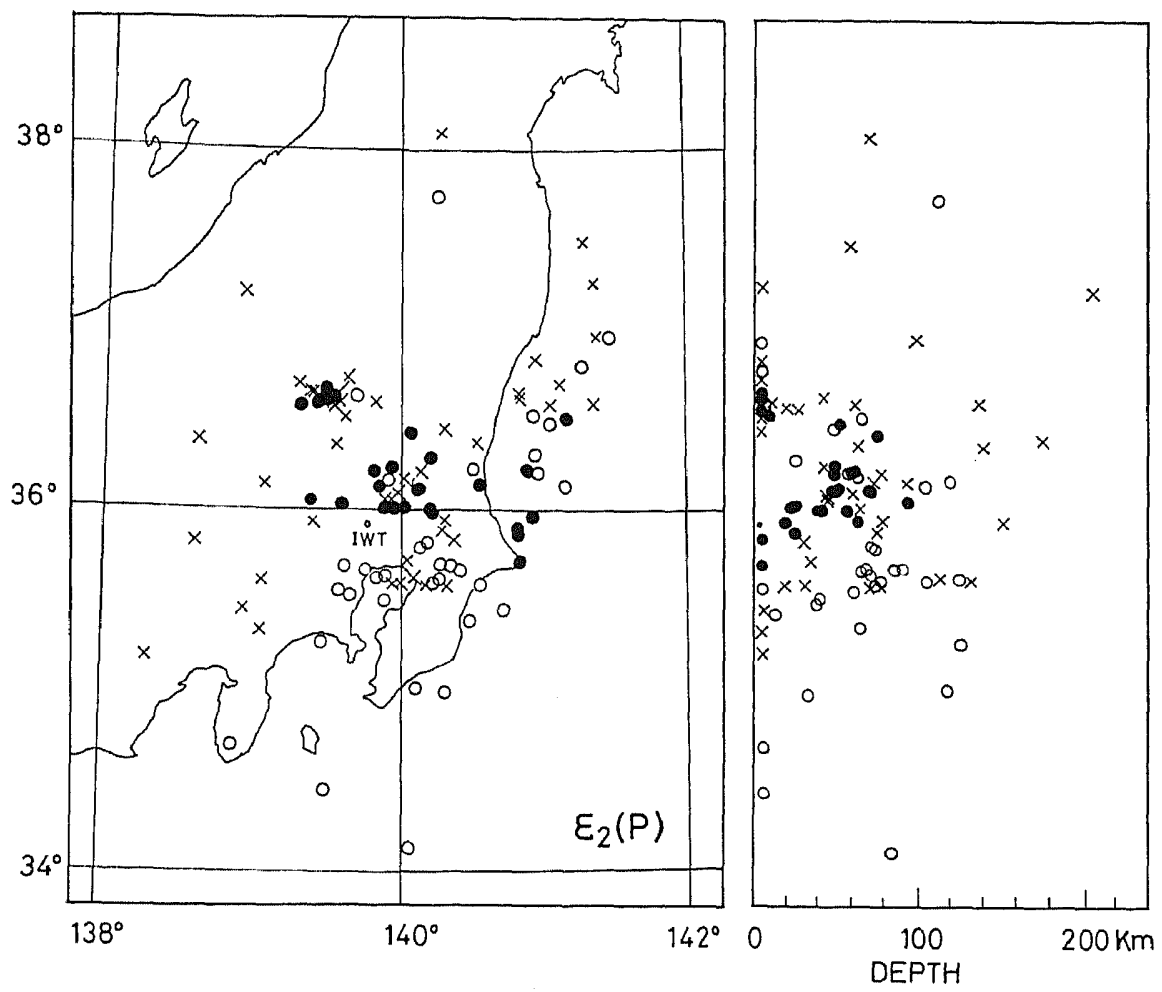

(b)

Fig. 5. Distribution of aspect ratios $\varepsilon_{1}$ and $\varepsilon_{2}$ for the P-wave particle motions. Time window $T$ is $0.8 \mathrm{sec}$. (a) Distribution of $\varepsilon_{1} . \quad 0,0.00-0.48 ; \times, 0.48-0.69 ; \bullet, 0.69$ 1.00. The hatched plane divides the Kanto district into two regions (see the text for the detail). (b) Distribution of $\varepsilon_{2}$. $0,0.00-0.22 ; \times, 0.22-0.38 ; 0.38-1.00$.

the direction of the principal axes of the trajectory ellipsoid. The representative model ellipsoid is shown in Fig. 8. It has a shape like an elliptic saucer: $\varepsilon_{1}\left(=\lambda_{2} / \lambda_{1}\right)=0.58$ and $\varepsilon_{2}\left(=\lambda_{3} / \lambda_{1}\right)=0.30$. The vector $a_{1}$ of the model ellipsoid points to the incident direction of the $\mathrm{P}$ wave, $\boldsymbol{a}_{2}$ is in a vertical plane and $\boldsymbol{a}_{3}$ in a horizontal plane. It is thus suggested that intensity of scattering is anisotropic. It is apparent that the intensity of scattering is higher in the vertical plane. This result is naturally expected, because the earth's crust is more heterogeneous in the vertical direction than in the horizontal direction. If the dimension of heterogeneity is smaller than the mean wave length amounting to some hundreds of meters, the scattering is equivalent to the Rayleigh scattering for the light, and frequency dependence of the scattering is consistent with that in the scattering theory (YAMAKAWA, 1956).

Examining Fig. 6 in more detail, we can find anisotropic patterns for $\boldsymbol{b}_{1}$ and $\boldsymbol{b}_{3}$ which seem to have an approximately symmetric axis along the line $a-b$. 


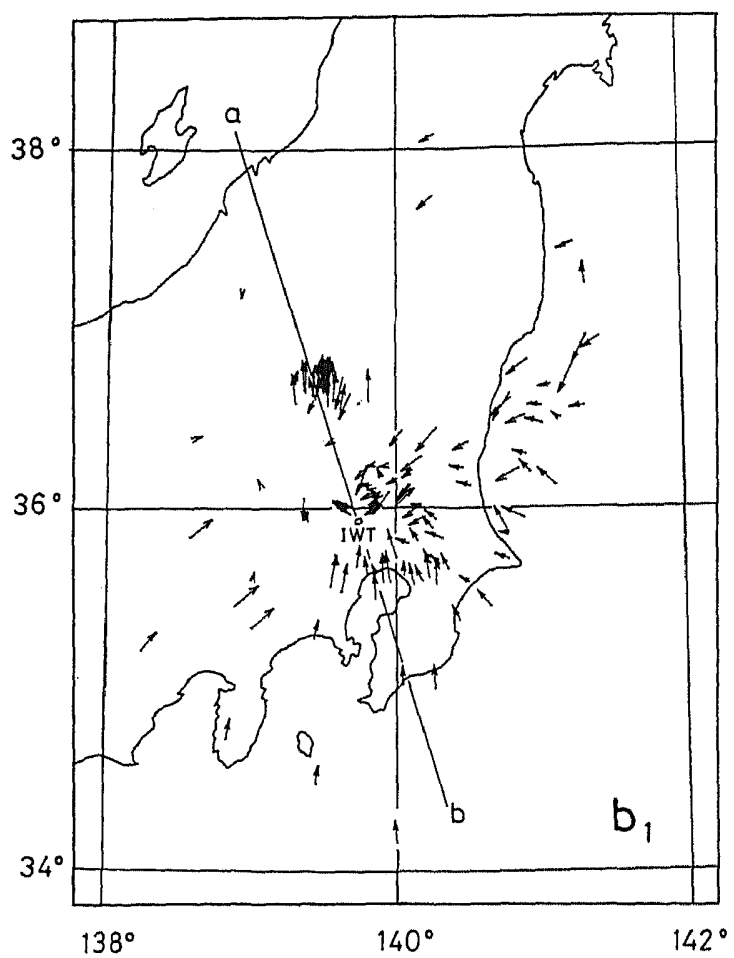

Fig. 6 (a)

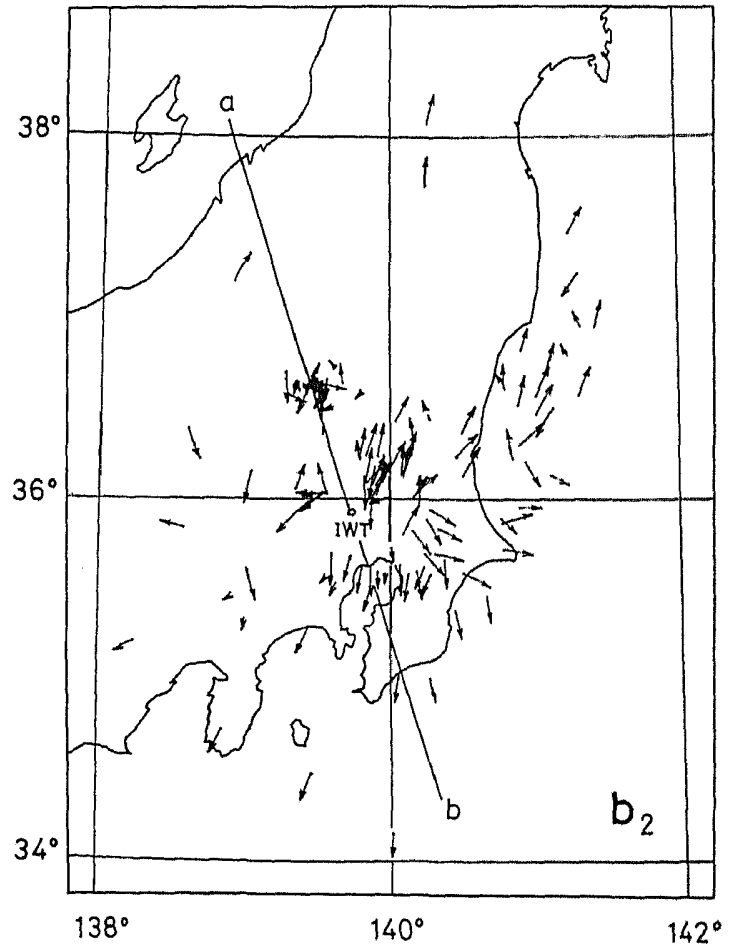

Fig. 6 (b) 


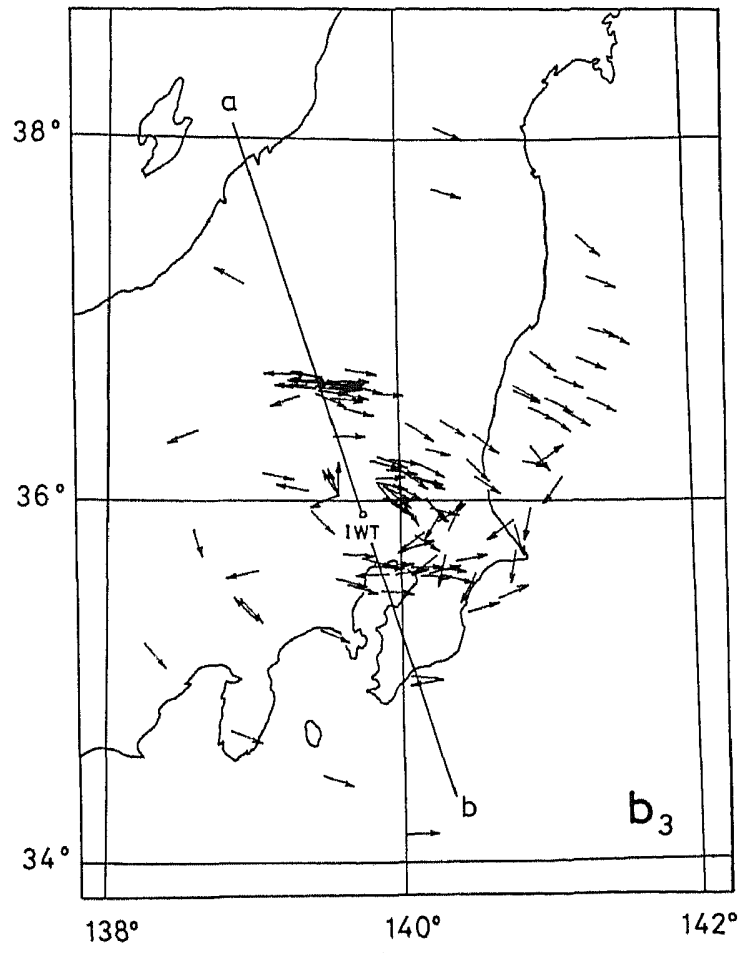

(c)

Fig. 6. Horizontal projections of eigenvectors of the P-wave particle motions for a time window of $0.8 \mathrm{sec}$. The origin of the vectors are moved from IWT to the epicenters. (a) $b_{1}$ (projection of $a_{1}$ ), (b) $b_{2}$ (projection of $a_{2}$ ), (c) $b_{3}$ (projection of $a_{3}$ ).

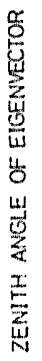
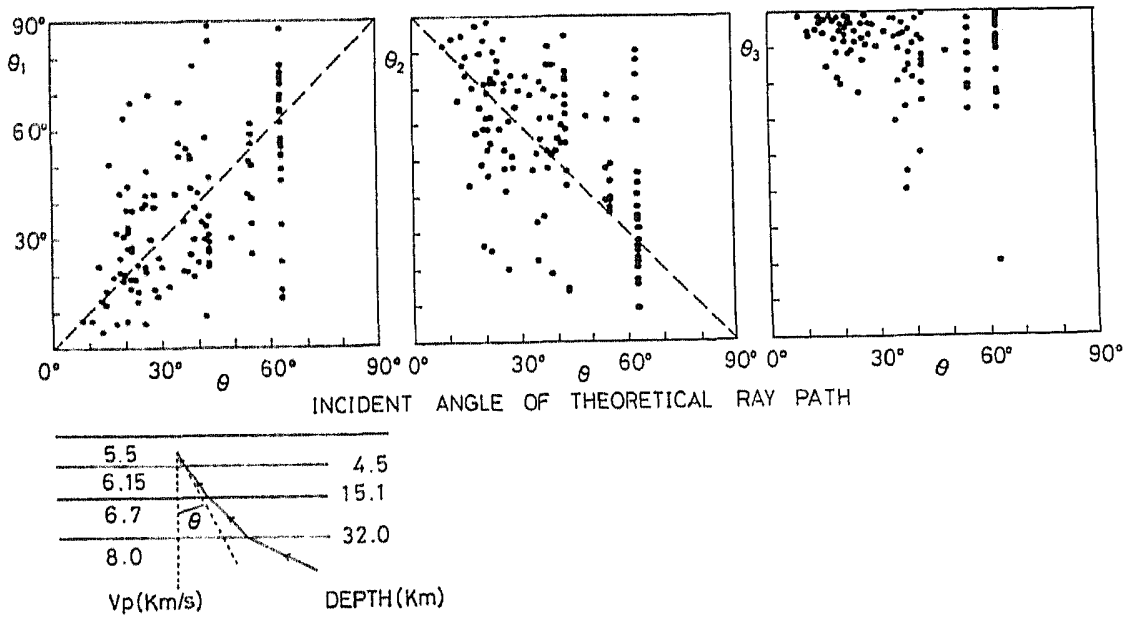

Fig. 7. Plots of zenith angles $\theta_{1}, \theta_{2}$ and $\theta_{3}$ of eigenvectors for the $P$-wave particle motions vs. theoretical incident angle $\theta$ based on the Mrkumo's crustal (1966) structure model (E-3A3), which is also shown in the figure. 


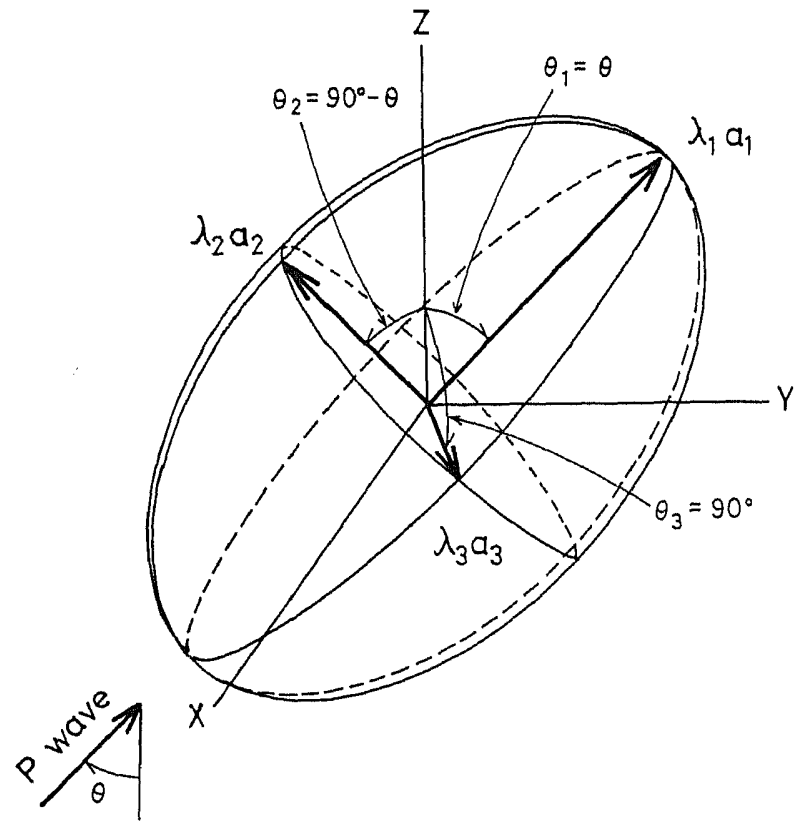

Fig. 8. The typical ellipsoid model for P-wave particle motion. The maximum axis $\lambda_{1} a_{1}$ points to the incident direction of the wave, the intermediate axis $\lambda_{2} a_{2}$ is in the vertical plane and the minimum axis $\lambda_{3} a_{3}$ in the horizontal plane. Aspect ratios are estimated as $\varepsilon_{1}\left(=\lambda_{2} / \lambda_{1}\right)=0.58$ and $\varepsilon_{2}\left(=\lambda_{3} / \lambda_{1}\right)=0.30$, for a time window of $0.8 \mathrm{sec}$.

Deflections $\delta$ for $b_{1}$ and $b_{3}$ from a radial pattern are plotted against azimuths $\varphi$ measured from IWT in Figs. 9(a) and (b), respectively. A systematic tendency characterized by boundaries of $\varphi=-20^{\circ}$ and $\varphi=160^{\circ}$ is found. Most of $\delta$ are positive for $\boldsymbol{b}_{1}$ and negative for $\boldsymbol{b}_{3}$ in the range of $\varphi=-20^{\circ}-160^{\circ}$, and vice versa in the other range of $\varphi$, though there are some exceptions. Such a tendency is reflected in Figs. 6(a) and (c) as the following arrow patterns; on the east side of the line $a-b$, most of $b_{1}$ are deflected closkwise from the direction towards IWT and on the west side counter-clockwise. Most of $\boldsymbol{b}_{3}$ on the east side tend to be expressed by a swirl clockwise around IWT, and on the west side counter-clockwise. Such a pattern may well be explained by introducing a rotation of vectors as illustrated in Fig. 10, where the isotropic vector patterns and those rotated in the vertical plane containing the line $\mathrm{a}-\mathrm{b}$ are shown for $\boldsymbol{a}_{1}, \boldsymbol{a}_{2}$ and $\boldsymbol{a}_{3}$ of the model ellipsoids. It is recognized that the anisotropic patterns of $b_{1}$ and $b_{3}$ in Figs. 6(a) and (c) are very similar to the rotated ones shown in Fig. 10. Therefore, an inverse rotation of the original pattern is expected to give the isotropic patterns for $\boldsymbol{b}_{1}$ and $\boldsymbol{b}_{3}$. In Fig. 11, histograms of the deflections $\delta$ of $\boldsymbol{b}_{1}$ are shown for rotation angles $\omega$ of $0^{\circ}, 10^{\circ}, 20^{\circ}$ and $30^{\circ}$. Among these, the rotation specified by $10^{\circ}$ is selected as the best fit of the radially isotropic pattern. In order to explain the source of such a rotation, either an instrumental effect or a crustal 


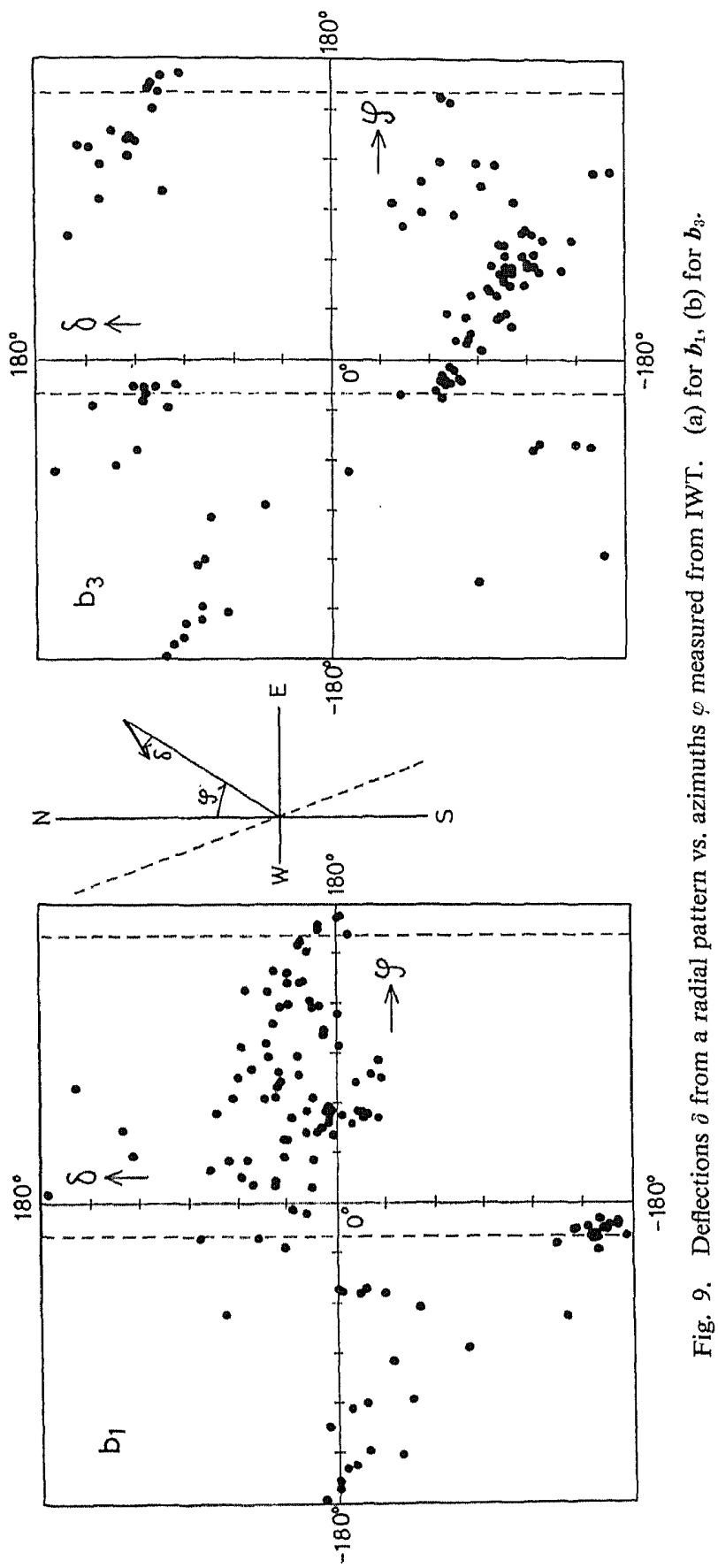




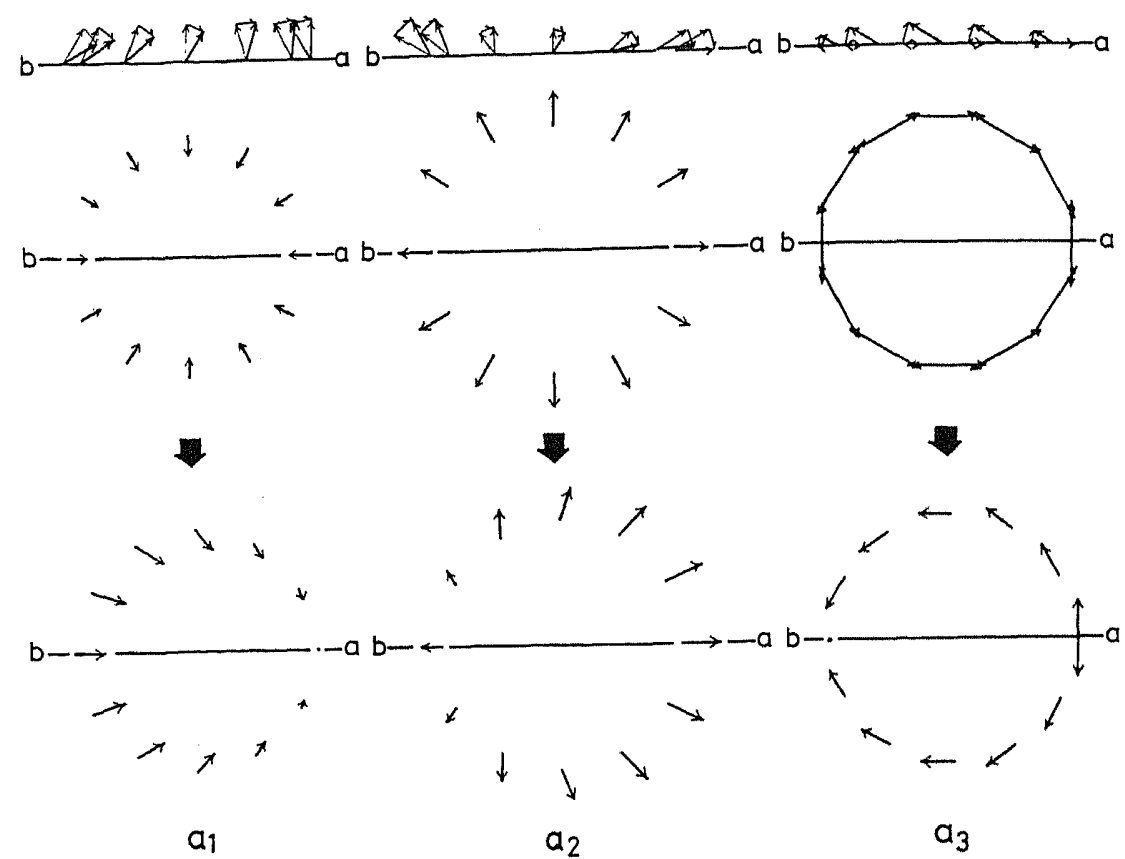

Fig. 10. Illustration explaining the deflection of vectors by a rotation. Isotropic model patterns for $a_{1}, a_{2}$ and $a_{3}$ are shown in the middle figures and those rotated in the vertical plane containing the line $a-b$ are shown in the bottom ones. The top figures are vertical sections, in which the vectors are rotated clockwise.

structure may be taken into consideration. As the source due to the instrumental effect, the following two possibilities may be considered: possible inclination of the seismometers and crosstalks of signals between the vertical component and the horizontal ones. However, it is difficult to attribute the source of the rotation to these factors because the seismometers keep the vertical with an accuracy of $1^{\circ}$ and the crosstalks are less than $2 \%$, which are too small to affect the result. Thus a dipping crustal structure is a more likely source of this rotation. We propose that the layered media are inclined about $10^{\circ}$, dipping to $\mathrm{N} 20^{\circ} \mathrm{W}$, the strike of $a-b$ as illustrated in Fig. 12. But from the data observed only at IWT, areal extent of such an inclined structure cannot be determined. For example, even a local anomaly in the vicinity of IWT is enough to explain the phenomena described above. A distribution of basement depth in the Kanto district is given by FUKUDA (1962), but this profile contradicts our result. Further investigation by using data obtained at other deep borehole observatories such as Shimohsa $\left(35.7934^{\circ} \mathrm{N}, 140.0238^{\circ} \mathrm{E},-2,277 \mathrm{~m}\right)$ or Fuchu $\left(35.6507^{\circ} \mathrm{N}, 139.4736^{\circ} \mathrm{E},-2,707 \mathrm{~m}\right)$ are necessary in order to ascertain the significance of the result.

In Fig. 6(a), a local anomaly is also seen in the neighborhood of Kobugahara $\left(36.7^{\circ} \mathrm{N}, 139.6^{\circ} \mathrm{E}\right)$, where an earthquake swarm was observed and most of $\boldsymbol{b}_{1}$ 


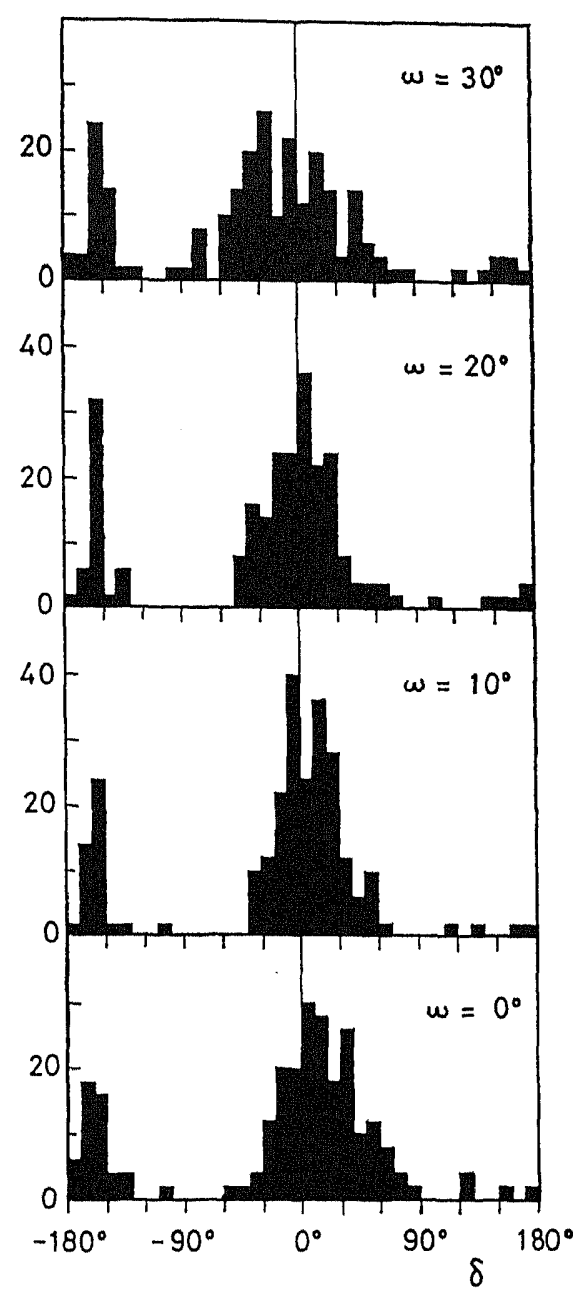

Fig. 11. Histograms of deflection $\delta$ of $b_{1}$ from a radial pattern for rotation angles $\omega$ of $0^{\prime \prime}, 10^{\circ}, 20^{\circ}$ and $30^{\circ}$.

point against IWT. It means that the seismic wave came from the upper side if $a_{1}$ is in the direction of the ray path. But such a ray path is not realistic for the velocity structure adopted here, even though the focal depths of these earthquakes are assumed to be very shallow. No reasonable explanation of this anomaly is given at the moment.

\section{$4.2 S$ wave}

The procedure similar to that for the $P$ wave was applied to the $S$ waves of 122 earthquakes. In the case of $S$ waves, directions of $\boldsymbol{a}_{1}$ and $\boldsymbol{a}_{2}$ and aspect ratio $\varepsilon_{1}$ are not unique because they are correlated to the polarization angle of the 


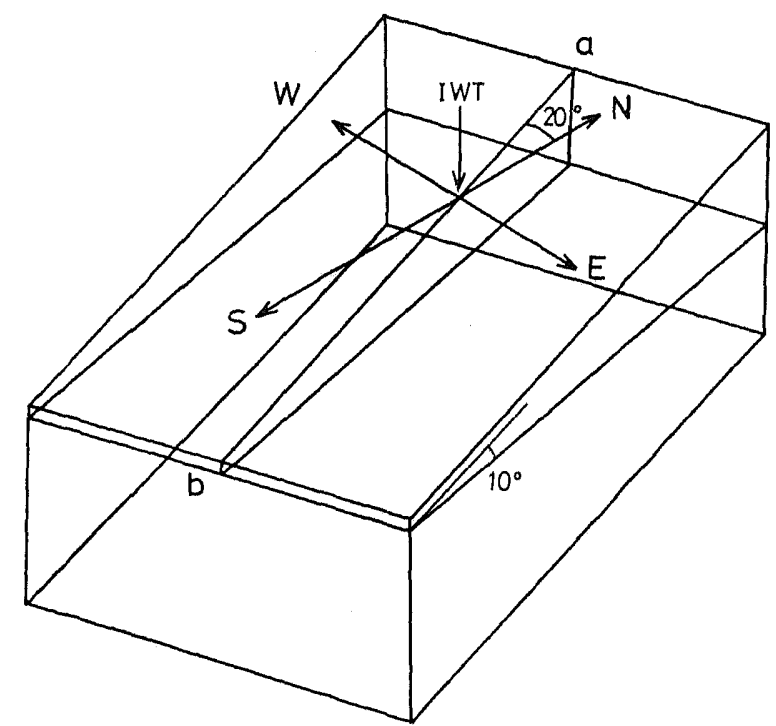

Fig. 12. A model of dipping crustal layers explaining the anisotropic pattern of the eigenvectors for the $\mathrm{P}$-wave particle motions. The layers dip $10^{\prime \prime}$ to $\mathrm{N} 20^{\prime} \mathrm{W}$, the strike of $a-b$.

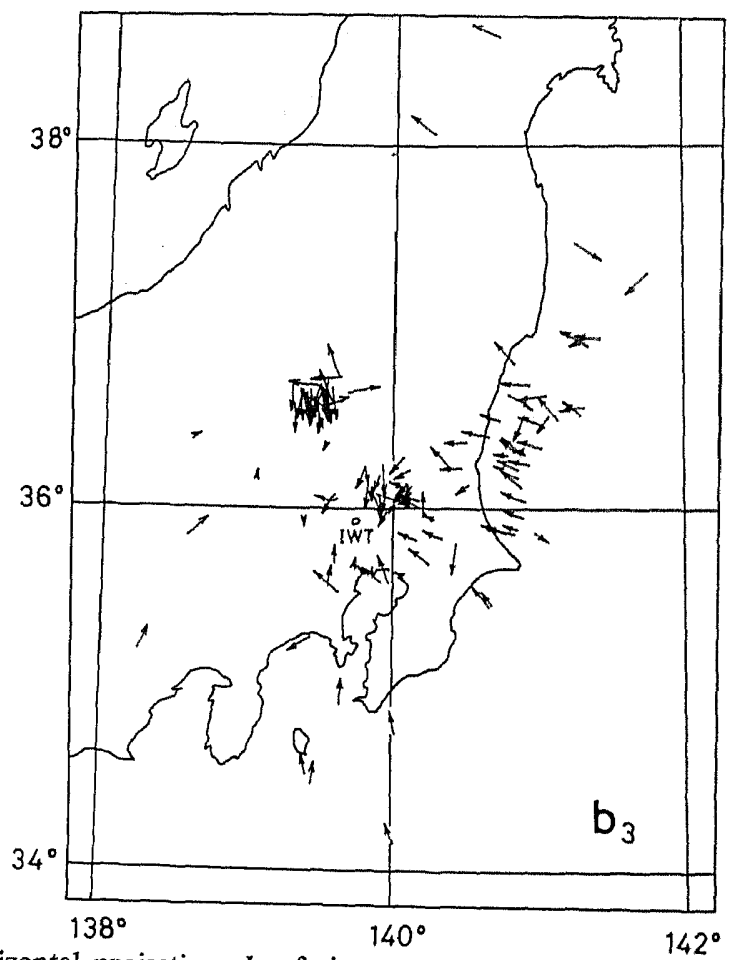

Fig. 13. Horizontal projections $b_{3}$ of eigenvectors $a_{3}$ of the S-wave particle motions for a time window of $0.8 \mathrm{sec}$. These projections are corrected with a rotation of $10^{\circ}$ according to the result obtained for the $\mathrm{P}$ wave. 


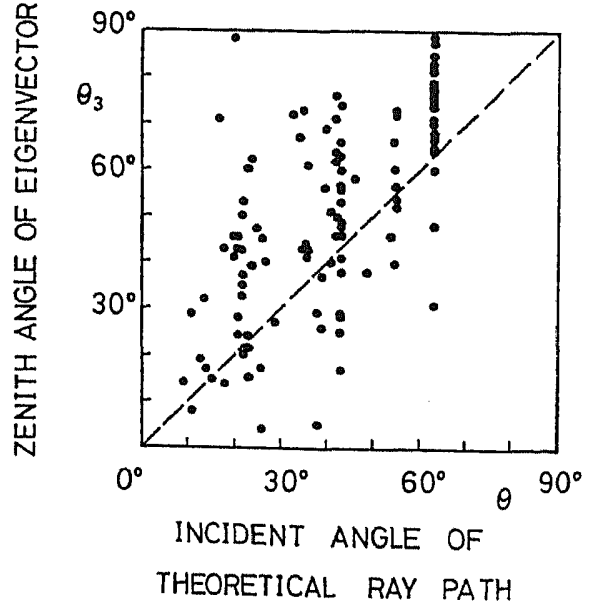

Fig. 14. Plots of zenith angles $\theta_{3}$ of eigenvectors for the S-wave particle motions vs. theoretical incident angle $\theta$ for the $\mathrm{P}$ wave.
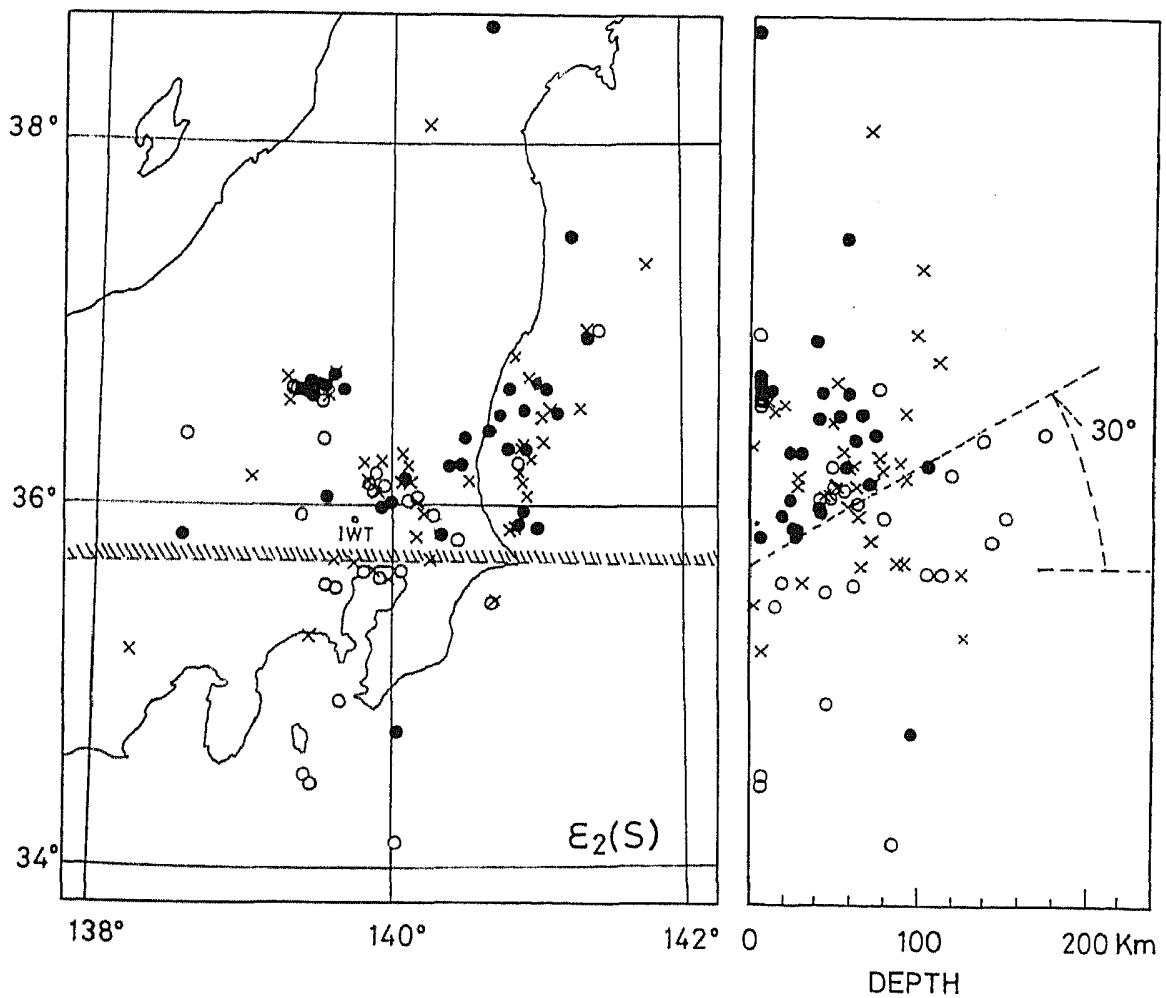

Fig. 15. Distribution of aspect ratios $\varepsilon_{2}$ for the $S$-wave particle motions. The time window $T$ is $0.8 \mathrm{sec}$. $0,0.00-0.38 ; \times, 0.38-0.58 ; 0,0.58-1.00$. The hatched plane divides the Kanto district into two regions (see the text for the detail). 
wave and strongly dependent on the earthquake mechanism. On the other hand, vectors $a_{3}$ are roughly in the direction of theoretical ray path as shown in Figs. 13 and 14 (they are corrected by using the same rotation as the $\mathrm{P}$ wave). This result may be understood by presuming an oblate spheroid, the flat face of which is perpendicular to the incident direction of the wave.

The regional distribution of aspect ratio $\varepsilon_{2}$ is shown in Fig. 15 in a manner similar to that of the $\mathrm{P}$ wave. It resembles that of $\varepsilon_{1}$ in the case of the $\mathrm{P}$ wave. A boundary striking in E-W direction along the latitude line of $35.7^{\circ} \mathrm{N}$ and inclined $30^{\circ}$ towards the north (hatched plane) can be drawn, dividing the Kanto district into two parts: a region north of the boundary of larger $\varepsilon_{2}$ and that south of the boundary of smaller $\varepsilon_{2}$. The mean value of dominant frequencies of $S$ waves is estimated as $8.5 \mathrm{~Hz}$ from Fig. 4 , but $9.0 \mathrm{~Hz}$ for those, of which $\varepsilon_{2}$ is more than $m+2 / 3 \sigma$, and $7.2 \mathrm{~Hz}$ for those, of which $\varepsilon_{2}$ is less than $m-2 / 3 \sigma$. This result also supports the conclusion obtained for the $\mathrm{P}$ wave.

\section{Conclusions}

Introducing the concept of trajectory ellipsoid, we attempted to express three-dimensional particle motions for the initial portions of $\mathrm{P}$ and $\mathrm{S}$ waves by an ellipsoidal approximation. The new technique enables us to represent the characteristics of $\mathrm{P}$ - and S-particle motions by such parameters as aspect ratios and eigenvectors. On applying the trajectory ellipsoid method to the data obtained at the Iwatsuki deep-borehole observatory, the following conclusions were resulted.

(1) The major axis of the trajectory ellipsoid for $\mathrm{P}$ waves points to the incident direction of the wave. Two other principal axes lie in the directions of $\mathrm{SV}$ and SH waves. Scattering of seismic waves mainly arises at the horizontal boundaries of the underground layers because the length of the SV axis is larger than that of the $\mathrm{SH}$ axis by a factor of nearly 2 .

(2) Nearby the latitude line of $36^{\circ} \mathrm{N}$, there exists a sharp boundary of which the northern side is characterized by larger values of $\varepsilon_{1}$ for $\mathrm{P}$ waves and $\varepsilon_{n}$ for $\mathrm{S}$ waves compared with those in the southern side. It suggests that the scattering of the wave is more intense in the northern region than in the southern region.

(3) The direction of the eigenvectors appears to coincide to the direction towards the epicenter provided they are rotated by about $10^{\circ}$ in the vertical plane including the line a-b. Such a fact may be attributed to a possible dip of the layers at unknown depths as shown in Fig. 12.

(4) In the case of $S$ waves, the characteristics of the trajectory ellipsoid are reflected in $\boldsymbol{a}_{3}$ and $\varepsilon_{2}$. Such characteristics are manifested in the patterns of $\boldsymbol{a}_{1}$ and $\varepsilon_{1}$, respectively, for $\mathrm{P}$ waves.

I am thankful to Mr. H. Sato for invaluable discussions about the scattering phenomenon of seismic waves, and to Mr. M. Takahashi and Dr. K. Tsumura for providing me with the 
data of earthquakes. I also acknowledge help from Miss Y. Katsuyama in data processing for AD conversion. Discussions with Dr. M. Ohtake were of great value.

\section{REFERENCES}

FUKUDA, O., Test boring at the Kasukabe region in the southern part of Kwanto district, Geol. Mon., 100, 1-16, 1962.

Furuzawa, T., Some problems of seismic data processing. Part 2. Data processing techniques for the detection and analysis of $\mathrm{P}$ and $\mathrm{S}$ waves of local earthquakes, Bull. Disas. Prev. Res. Inst., Kyoto Univ., 24, Part 3, No. 222, 127-145, 1974.

Furuzawa, T. and K. Irikura, The direction of the particle motions of local small earthquakes, Annu. Disas. Prev. Res. Inst., Kyoto Univ., 13A, 149-161, 1970.

Kurihara, K., T. Utsuno, and T. Nagamune, Deviation of directions of initial $\mathrm{P}$ waves at Tokatidake, Hokkaido, Japan, Q. J. Seismol., 39 (4), 75-81, 1974.

Mikumo, T., A study on crustal structure in Japan by the use of seismic and gravity data, Bull. Earthq. Res. Inst., Univ. Tokyo, 44, 965-1007, 1966.

NuTrur, O., The effect of the earth's surface on the S wave particle motion, Bull. Seismol. Soc. Am., 51, 237-246, 1961 .

Sato, H. and S. MATsumura, Three-dimensional analysis of scattered $\mathrm{P}$ waves on the basis of the PP single isotropic scattering model, J. Phys. Earth, 28, 521-530, 1980.

Tsujiura, M., Regional variation of micro-earthquake spectrum-Kanto district, Zisin $(J$. Seismol. Soc. Japan), Ser. 2, 26, 370-375, 1973.

UTsu, T., On deflection of the direction of initial motion of P wave, Q. J. Seismol., 21 (1), 13-20, 1956.

WadA, T. and K. Nishimura, A structure of the volcano Aso deduced from the azimuthal deviation of P-wave, Annu. Disas. Prev. Res. Inst., Kyoto Univ., 14A, 139-148, 1971.

YAMAKAWA, N., Investigation of the disturbance produced by spherical obstacles on the elastic waves. I. On the scattering of the elastic waves by a spherical obstacles, $Q$. J. Seismol., 21 (1), 1-12, 1956.

Yamamizu, F. and N. Goro, Direct measurement of seismic wave velocities in deep soil deposits, Proc. 5th Japan Earthq. Eng. Symp., pp. 329-336, 1978. 